\title{
Correction to: An Explainable Model for Fault Detection in HPC Systems
}

\author{
Martin Molan (D), Andrea Borghesi (D), Francesco Beneventi, \\ Massimiliano Guarrasi (D), and Andrea Bartolini (D)
}

\section{Correction to: \\ Chapter "An Explainable Model for Fault Detection in HPC Systems" in: H. Jagode et al. (Eds.): High Performance Computing, LNCS 12761, https://doi.org/10.1007/978-3-030-90539-2_25}

The chapter was inadvertently published with the spelling error in the first author's name. It has been corrected to "Martin Molan". 